\title{
Combination of surface- and interference-enhanced Raman scattering by CuS nanocrystals on nanopatterned Au structures
}

\author{
Alexander G. Milekhin ${ }^{* 1,2}$, Nikolay A. Yeryukov ${ }^{1,2}$, Larisa L. Sveshnikova ${ }^{1}$, \\ Tatyana A. Duda ${ }^{1}$, Ekaterina E. Rodyakina ${ }^{1,2}$, Victor A. Gridchin ${ }^{1,3}$, \\ Evgeniya S. Sheremet ${ }^{1,4}$ and Dietrich R. T. Zahn ${ }^{1,4}$
}

\author{
Full Research Paper \\ Address: \\ ${ }^{1}$ A. V. Rzhanov Institute of Semiconductor Physics, pr. Lavrentieva, \\ 13, Novosibirsk 630090, Russia, ${ }^{2}$ Novosibirsk State University, \\ Pirogov str. 2, Novosibirsk 630090, Russia, ${ }^{3}$ Novosibirsk State \\ Technical University, pr. Karl Marx, 20, Novosibirsk, 630092, Russia \\ and ${ }^{4}$ Semiconductor Physics, Technische Universität Chemnitz, \\ D-09107 Chemnitz, Germany

\section{Email:} \\ Alexander G. Milekhin* - milekhin@isp.nsc.ru \\ ${ }^{*}$ Corresponding author

\section{Keywords:} \\ copper sulfide (CuS) nanocrystals; interference-enhanced Raman \\ spectroscopy; phonons; surface-enhanced Raman spectroscopy \\ Beilstein J. Nanotechnol. 2015, 6, 749-754. \\ doi:10.3762/bjnano.6.77 \\ Received: 12 September 2014 \\ Accepted: 17 February 2015 \\ Published: 17 March 2015 \\ This article is part of the Thematic Series "Self-assembly of \\ nanostructures and nanomaterials". \\ Guest Editor: I. Berbezier \\ (C) 2015 Milekhin et al; licensee Beilstein-Institut. \\ License and terms: see end of document.
}

\begin{abstract}
We present the results of a Raman study of optical phonons in CuS nanocrystals (NCs) with a low areal density fabricated through the Langmuir-Blodgett technology on nanopatterned Au nanocluster arrays using a combination of surface- and interferenceenhanced Raman scattering (SERS and IERS, respectively). Micro-Raman spectra of one monolayer of CuS NCs deposited on a bare Si substrate reveal only features corresponding to crystalline Si. However, a new relatively strong peak occurs in the Raman spectrum of CuS NCs on Au nanocluster arrays at $474 \mathrm{~cm}^{-1}$. This feature is related to the optical phonon mode in CuS NCs and manifests the SERS effect. For CuS NCs deposited on a $\mathrm{SiO}_{2}$ layer this phonon mode is also observed due to the IERS effect. Its intensity changes periodically with increasing $\mathrm{SiO}_{2}$ layer thickness for different laser excitation lines and is enhanced by a factor of about 30. CuS NCs formed on Au nanocluster arrays fabricated on IERS substrates combine the advantages of SERS and IERS and demonstrate stronger SERS enhancement allowing for the observation of Raman signals from CuS NCs with an ultra-low areal density.
\end{abstract}

\section{Introduction}

Investigations of Raman scattering in nanostuctures such as nanocrystals (NCs) are limited by a low Raman cross-section because of the very low scattering volume of the nanostructures.
Surface-enhanced Raman spectroscopy (SERS) taking advantage of plasmonics leads to a remarkable increase of the Raman sensitivity as shown for several semiconductor NC types [1-13]. 
The SERS effect by longitudinal optical (LO) phonons of CdS in $\mathrm{Ag}-\mathrm{CdS}$ composite nanoparticles in solution and on a solid substrate was demonstrated [1,2]. Resonant SERS enhancement by LO phonons of CdSe was observed in core-shell $\mathrm{CdSe} / \mathrm{ZnS}$ NCs deposited on commercially available nanostructured Au substrates [3]. Later, the SERS effect by LO phonons in $\mathrm{CdSe}$ in $\mathrm{CdSe} / \mathrm{ZnS} \mathrm{NCs}$ was realized on non-ordered nanostructured Ag surfaces [4]. Very recently, Lee et al. [5] reported the observation of SERS by surface optical (SO) and LO phonon modes in a CdSe core and the transverse optical (TO) phonon mode in a $\mathrm{ZnS}$ shell of core-shell CdSe/ZnS NCs attached to the surface of a Au nanowire. The spectrum of optical and interface phonons was obtained from the analysis of SERS spectra of pure CdSe NCs, core-shell CdSe/CdS, and CdSe/CdZnS NCs deposited on Ag SERS substrates [6]. A prominent enhancement of Raman scattering by LO phonons was observed in $\mathrm{Au}-\mathrm{ZnO} \mathrm{NC}$ nanocomposites [7] and $\mathrm{ZnO}$ NCs covered by Ag [8] excited near resonance with the interband electronic transitions in $\mathrm{ZnO} \mathrm{NCs}$.

Anomalously enhanced Raman scattering by LO phonons in epitaxial $\mathrm{GaN}$ and $\mathrm{ZnO} \mathrm{NC}$ thin films covered with Ag was also explained by SERS [9]. A pronounced $10^{4}$-fold SERS enhancement by surface optical phonons was observed for $\mathrm{ZnO} \mathrm{NCs}$ excited in resonance with localised surface plasmon in $\mathrm{Ag}$ nanoclusters deposited on $\mathrm{ZnO} \mathrm{NCs}$ and out of the resonance $[10,11]$. SERS by LO phonons of CdTe was investigated in mixed Ag-CdTe NCs with a controllable Ag nanoparticle/CdTe $\mathrm{NC}$ mixture ratio [12]. The first report on the observation of the SERS effect by optical phonons in CuS NCs on ordered arrays of Au nanoclusters fabricated in a nanolithography process was published recently [13]. CuS NCs were synthesised by using the Langmuir-Blodgett (LB) technique, which allows the formation of arrays of $\mathrm{CuS} \mathrm{NCs}$ with variable areal density. It was established that the variation of Au nanocluster size and shape in the nanostructured Au arrays governs the local surface plasmon resonance energy enabling resonance SERS in absorbates deposited on the arrays [13-17]. Moreover, CuS NCs are resistant against intense laser excitation even under resonant conditions. This is important for micro-Raman experiments with the NCs on nanostructured Au arrays under excitation in the green spectral region. These two issues make $\mathrm{CuS}$ NCs an attractive model system for SERS investigations of inorganic analytes. As it was demonstrated in [18], extremely thin absorbing coatings deposited on an antireflection layer (typically $100 \mathrm{~nm} \mathrm{SiO} 2$ on $\mathrm{Si}$ ) exhibit interference-enhanced Raman scattering (IERS) thus providing an alternative opportunity to enhance the Raman response by phonons in absorbates.

In this paper we present the results of both IERS and SERS and their combination by optical phonons in $\mathrm{CuS}$ NCs deposited on metal and semiconductor structures aiming at a maximal enhancement of the phonon response from $\mathrm{CuS}$ NCs.

\section{Results and Discussion Conventional Raman scattering}

A typical Raman spectrum of a dense array of $\mathrm{CuS}$ NCs deposited by LB technology on a Au substrate is shown in Figure 1. The average thickness of CuS NC film is about $32 \mathrm{~nm}$, which corresponds to 5-6 monolayer (ML) coverage by $\mathrm{CuS}$ NCs. The spectrum reveals a pronounced peak at $474 \mathrm{~cm}^{-1}$, which is assigned to vibrational (stretching) modes from the covalent S-S bonds [19] and a much weaker peak at about $270 \mathrm{~cm}^{-1}$ attributed to the $\mathrm{Cu}-\mathrm{S}$ bond vibration [19]. Therefore, the main attention was paid to the analysis of the Raman intensity of the most intense mode at $474 \mathrm{~cm}^{-1}$.

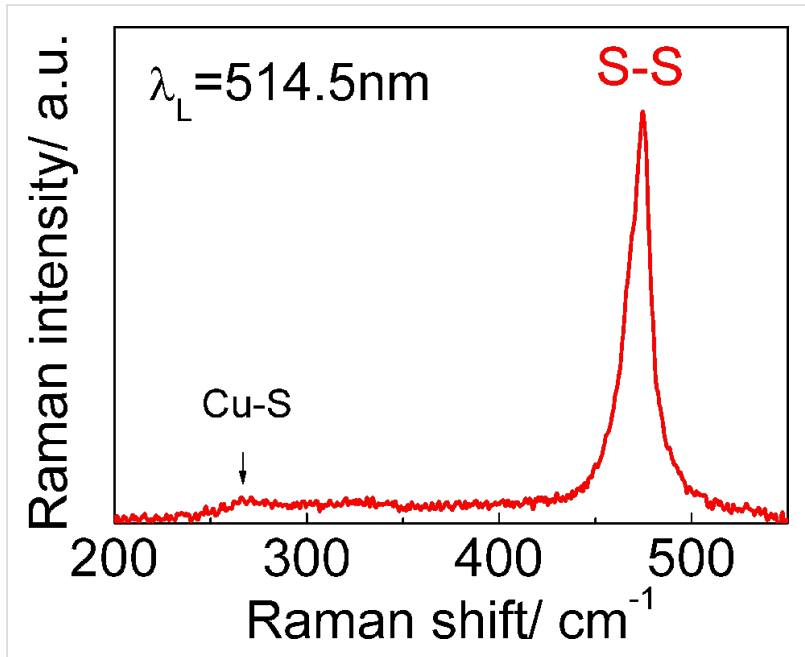

Figure 1: A typical Raman spectrum of the dense ensemble of CuS NCs (about 5-6 MLs) on a Au substrate excited with $514.5 \mathrm{~nm}$.

\section{Interference-enhanced Raman scattering}

It was already established that thickness and refractive index of the $\mathrm{SiO}_{2}$ layer determine conditions for laser light interference, and thus for IERS by absorbates deposited on the sample surface $[18,20]$. Since these two parameters are crucial for the enhancement of the Raman scattering by the absorbing NC layer, we used spectroscopic ellipsometry to determine the precise value of the $\mathrm{SiO}_{2}$ layer thickness that gives the maximal IERS signal. The data on optical properties of $\mathrm{CuS} \mathrm{NC}$ layer are also absent in literature, therefore, a wedge-shaped $\mathrm{SiO}_{2}$ layer with the thickness varying in the range from 0 to $570 \mathrm{~nm}$ was fabricated. This sample served as a substrate for the deposition of CuS NCs by using the LB technique and for further Raman experiments.

The micro-Raman scattering study was performed in a backscattering geometry using a moving stage for precise posi- 
tioning along the wedge-shaped sample in the direction of increasing thickness. Figure 2 shows the Raman spectra of $\mathrm{CuS}$ $\mathrm{NCs}$ recorded from areas with different $\mathrm{SiO}_{2}$ thickness. A significant periodic enhancement of the Raman scattering intensity by the phonon mode of CuS NCs indicates the presence of the IERS phenomenon. As the interference conditions inside the transparent oxide layer for the incident light wavelength changes with oxide thickness, a noticeable enhancement of the Raman scattering by the Si phonon (about 30\%) also takes place. The periodic variation of the enhancement factor of the phonon mode in $\mathrm{CuS} \mathrm{NCs}$ as a function of $\mathrm{SiO}_{2}$ layer thickness (Figure 3) was established for different laser excitation lines $(632.8,514.5$, and $325 \mathrm{~nm})$. Here, the intensity of the phonon mode of $\mathrm{CuS}$ NCs on a bare Si should be taken as a reference. However, it is worth mentioning that the intensity of the phonon

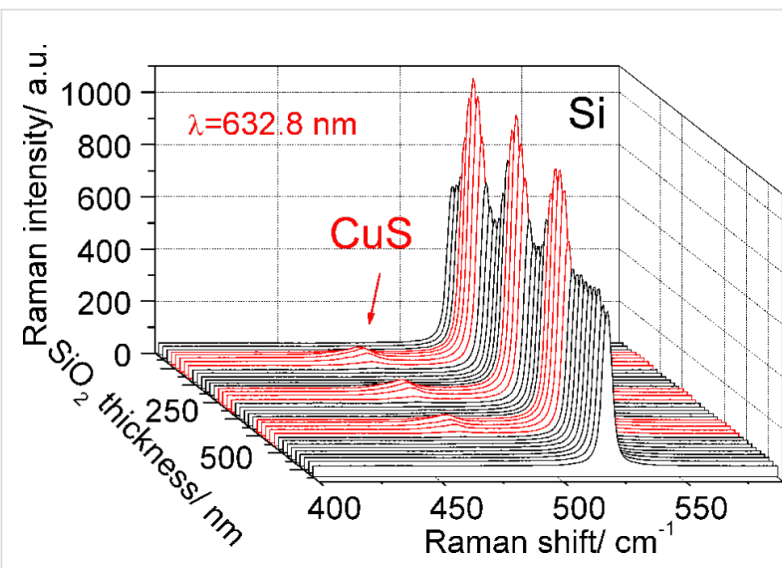

Figure 2: Raman spectra of CuS NCs (of about $1 \mathrm{ML}$ coverage) fabricated on a $\mathrm{Si}$ substrate with a $\mathrm{SiO}_{2}$ layer of variable thickness. Raman spectra were taken using $514.5 \mathrm{~nm}$ excitation wavelength. mode is equal or below the noise level. Therefore, only an estimate of the IERS (as well as SERS) enhancement factor is possible. The maximal estimated IERS enhancement for the excitation wavelength of $632.8 \mathrm{~nm}$ reaches a value of at least 30 for a $\mathrm{SiO}_{2}$ layer thickness of $75 \mathrm{~nm}$. As expected, the IERS enhancement maximum is observed for thicker $\mathrm{SiO}_{2}$ layers with increasing laser excitation wavelength. This $\mathrm{SiO}_{2}$ layer thickness $(75 \mathrm{~nm})$ was used for further combined IERS and SERS experiments.

\section{Surface-enhanced Raman scattering}

A typical SEM image of the edge of a periodic Au nanocluster array on a Si substrate with deposited CuS NCs is presented in Figure 4a. One can see that the CuS NCs are homogeneously distributed on the $\mathrm{Au}$ nanocluster array and on the bare $\mathrm{Si}$

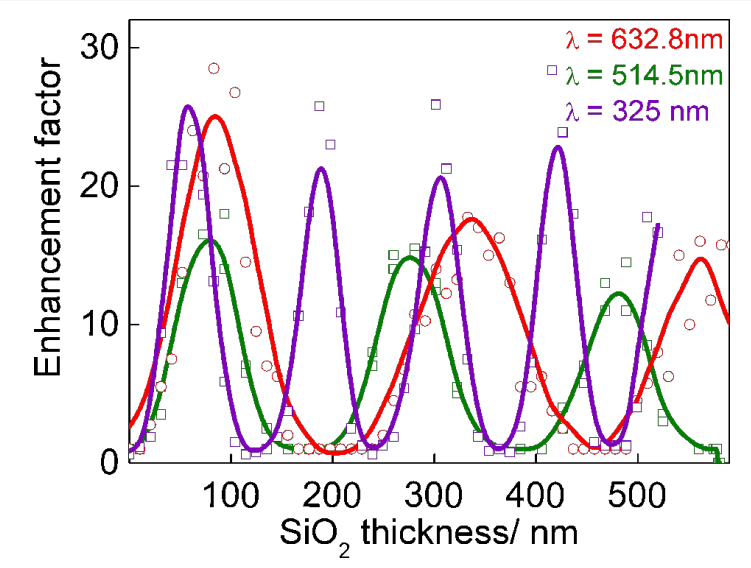

Figure 3: The dependence of the IERS enhancement factor of phonon modes in CuS NCs on the thickness of the $\mathrm{SiO}_{2}$ layer determined for laser excitation lines at $632.8,514.5$, and $325 \mathrm{~nm}$.

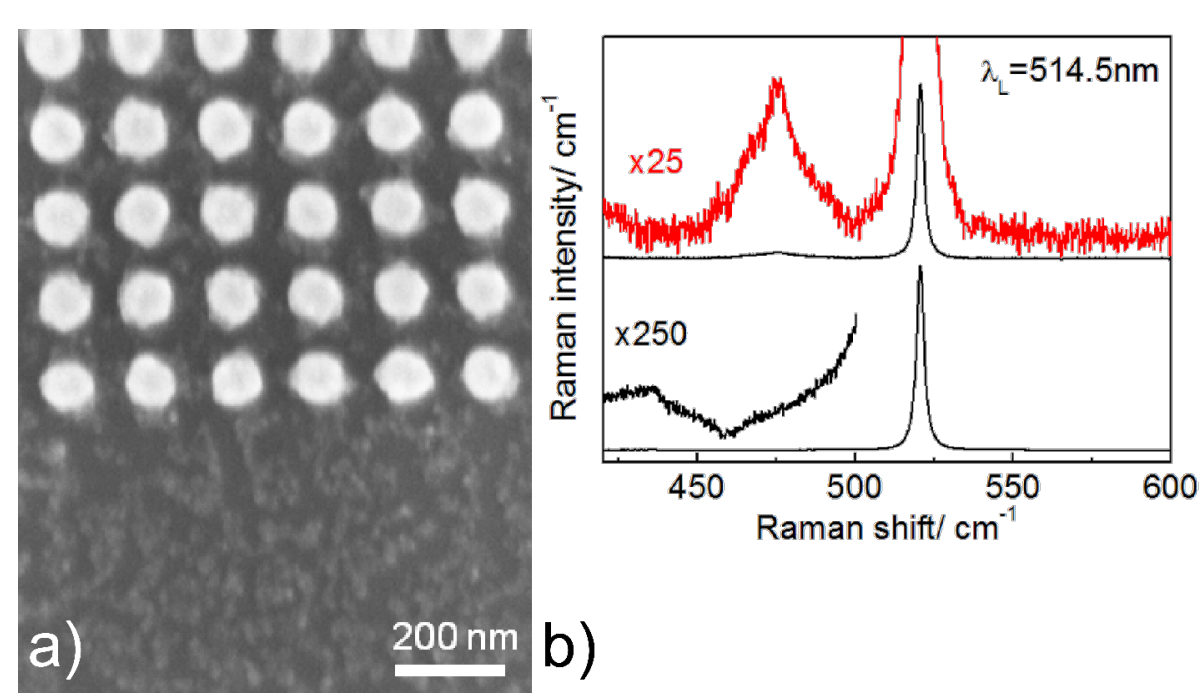

Figure 4: a) SEM image and b) micro-Raman spectra of CuS NCs deposited on Si (lower part) and Au nanocluster array (upper part). 
surface with an average thickness of about 1 ML. The Raman spectrum measured from the area where CuS NCs are formed on bare Si (lower part in Figure 4a) shows only one strong Raman line at $521 \mathrm{~cm}^{-1}$ related to the Si substrate (lower curve in Figure 4b). Weak Raman features located in the spectral range of $400-500 \mathrm{~cm}^{-1}$ are also typical for monocrystalline $\mathrm{Si}$ [21] while no evidence of the phonon modes from $\mathrm{CuS} \mathrm{NCs}$ is detected. However, a strong Raman band centred near $474 \mathrm{~cm}^{-1}$ arises when the Raman spectra are acquired from the area where $\mathrm{CuS}$ NCs are deposited on the nanocluster array. The SERS enhancement factor can hardly be determined since no reference signal from $\mathrm{CuS}$ NCs is detected on bare Si. However, depending on the NC areal density the SERS enhancement factor was estimated as $30-50$, which is comparable to that of IERS.

\section{Combination of SERS and IERS}

In order to achieve even stronger enhancement of Raman scattering by phonons in CuS NCs by both IERS and SERS, CuS NCs were deposited on arrays of Au nanoclusters fabricated using nanolithography on a $75 \mathrm{~nm}$ thick $\mathrm{SiO}_{2}$ layer. Obviously, the LSPR energy of the Au nanocluster arrays fabricated on a $\mathrm{SiO}_{2}$ layer and on a $\mathrm{Si}$ substrate can be different due to the difference of dielectric functions of $\mathrm{SiO}_{2}$ and $\mathrm{Si}[22,23]$ that determine the LSP energy. Indeed, the CuS NCs on $\mathrm{Au}$ nanocluster arrays fabricated on a Si substrate excited with $514.5 \mathrm{~nm}$ demonstrate a prominent SERS signal from optical phonons of CuS NCs with the same effective coverage (of about $1 \mathrm{ML}$ ) (Figure 4), while no signal is detected when Au nanoclusters were fabricated on $\mathrm{SiO}_{2}$ layer (not shown here). Most probably the shift of the LSPR energy in Au arrays fabricated on $\mathrm{SiO}_{2}$ layer is responsible for the absence of $\mathrm{CuS}$ phonon modes in Raman spectra excited with $514.5 \mathrm{~nm}$. The situation changes when the Raman scattering of the same structures was investigated under excitation with a red laser line $(632.8 \mathrm{~nm})$. CuS NCs on Au nanocluster arrays fabricated on Si exhibit noticeable SERS signal with an intensity comparable with that of the IERS signal when CuS NCs are deposited on a bare $75 \mathrm{~nm}$ thick $\mathrm{SO}_{2}$ layer or an array of $\mathrm{Au}$ (Figure 5, curves 2 and 3, respectively). Note, that again no Raman signal is detected for NCs deposited on a bare Si substrate (Figure 5, curve 1). However, CuS NCs on Au nanocluster arrays fabricated on a $\mathrm{SiO}_{2}$ layer (Figure 5, curve 4) reveal further significant enhancement of Raman scattering (a factor of about 6). According to [24] the electromagnetic field has a maximum in the vicinity of an adsorbate/oxide interface due to constructive interference. The SERS intensity is proportional to the forth order of electromagnetic field and, therefore, can be significantly enhanced for the adsorbate (or CuS NCs) located in the local field. The constructive interference in oxide layers was successfully used for achieving the maximum field enhance- ment for optical antennas [25], for realising the effect of co-enhanced IERS and SERS by organic molecules [26] and graphene [27], as well as for designing a chip for single molecular detection [28]. Thus, the use of the combination of SERS and IERS is preferable for the detection of a weak Raman response from a tiny amount of a material such as $\mathrm{CuS}$ NCs of an ultra-low areal density.

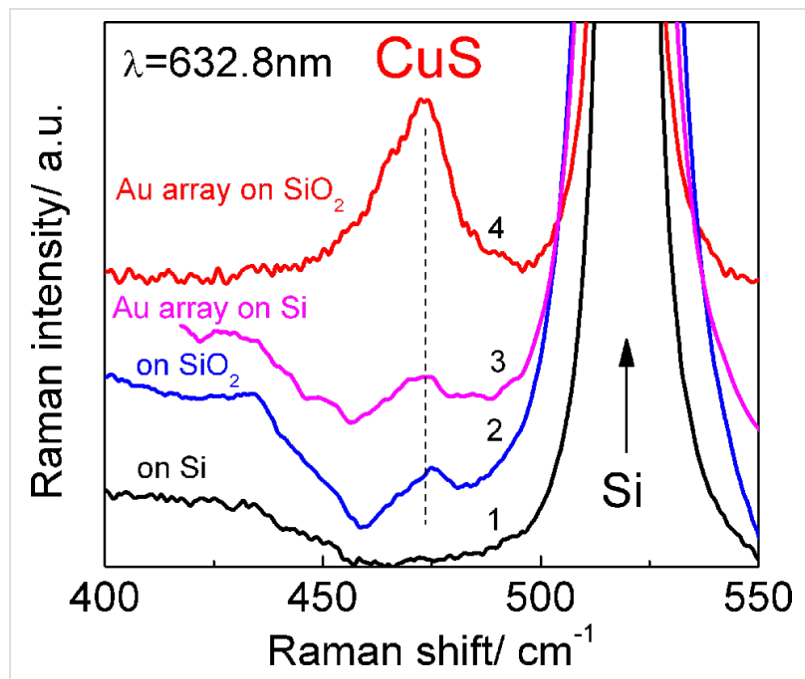

Figure 5: Raman spectra of CuS NCs deposited on bare Si, $75 \mathrm{~nm}$ $\mathrm{SiO}_{2}$ layer on $\mathrm{Si}$, and on $\mathrm{Au}$ arrays fabricated on $\mathrm{Si}$ and $\mathrm{SiO}_{2}$ layer on Si measured with $632.8 \mathrm{~nm}$.

Figure 6 shows a SEM image of homogeneously deposited $\mathrm{CuS}$ NCs with an areal density about ten times lower than that determined for the sample presented in Figure 4a. The SERS spectrum of the CuS NCs with ultra-low areal density shows a Raman phonon response at the same frequency position (about $474 \mathrm{~cm}^{-1}$ ) with an intensity about 25 times weaker than for $1 \mathrm{ML}$ of CuS NCs. This low intensity is explained by the smaller number of CuS NCs taking part in the SERS process. It is important to emphasise that the full width at half-maximum (FWHM) decreases from 17 to $9 \mathrm{~cm}^{-1}$ with decreasing areal density. This can be explained by the decreasing interaction between individual $\mathrm{CuS}$ NCs in the ensembles with the ultralow areal density and by the reduced number of agglomerates of CuS NCs.

\section{Conclusion}

A periodical enhancement of Raman scattering by optical phonons from one monolayer of $\mathrm{CuS}$ nanocrystals fabricated by the Langmuir-Blodgett technology on $\mathrm{SiO}_{2}$ layer deposited on a $\mathrm{Si}$ substrate was observed with the variation of the $\mathrm{SiO}_{2}$ layer thickness due to interference-enhanced Raman scattering. The pronounced enhancement of the Raman scattering from the nanocrystal ensembles deposited on arrays of Au nanoclusters evidences the surface enhanced Raman scattering effect. The 

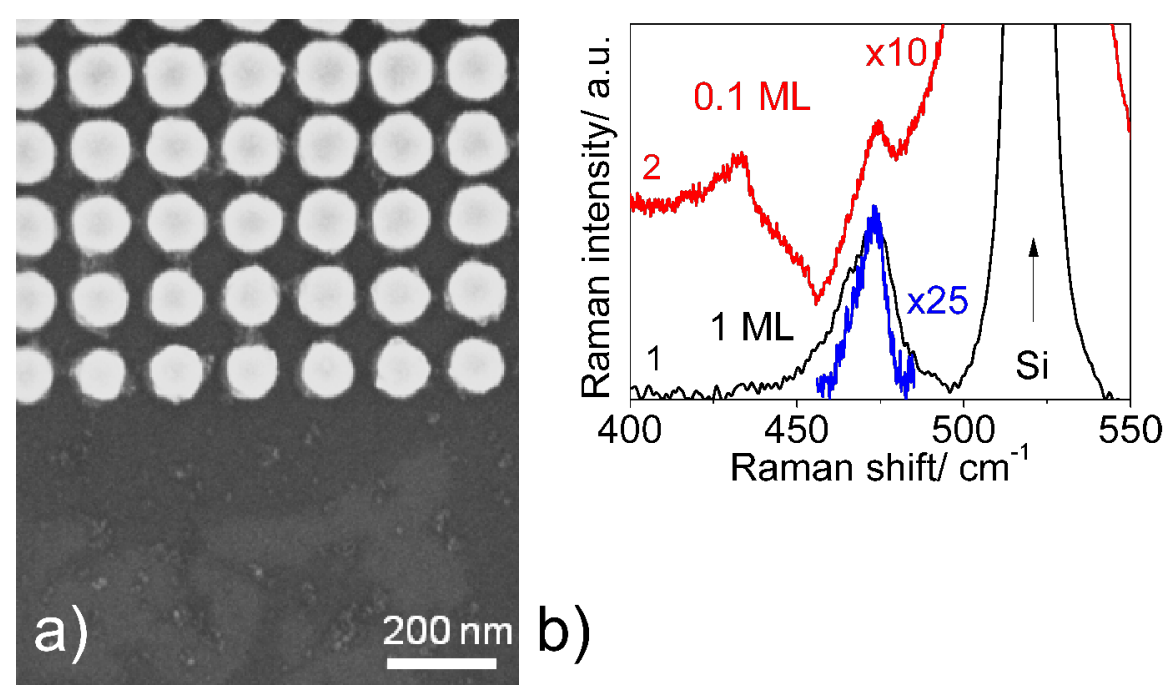

Figure 6: a) SEM image of CuS NCs with an ultra-low areal density deposited on Au arrays on $\mathrm{SiO}_{2}$ layer and b) SERS-IERS Raman spectra measured with $632.8 \mathrm{~nm}$. SERS spectrum of CuS NCs with $1 \mathrm{ML}$ coverage on Au arrays formed on $\mathrm{SiO}_{2}$ layer (curve 1) together with the Raman response of the CuS NCs of ultra-low areal density (denoted as $0.1 \mathrm{ML}$, curve 2). The 25 times enlarged fragment of curve 2 is shown for comparison.

combination of interference- and surface-enhanced Raman scattering led to a stronger enhancement of the phonon response by a factor of at least 180 , and was successfully applied for probing the phonon spectrum of $\mathrm{CuS}$ nanocrystals with an ultra-low areal density.

\section{Experimental}

The layer of thermally grown $\mathrm{SiO}_{2}$ with gradually varying thickness (from 0 to $570 \mathrm{~nm}$ ) was prepared on a Si substrate in a wet chemical process by controlled dipping of a Si substrate covered by a homogeneous $600 \mathrm{~nm}$ thick $\mathrm{SiO}_{2}$ layer into HF solution in $\mathrm{H}_{2} \mathrm{O}$ (with bulk ratio 2:5) and served as IERS substrate. The thickness of the $\mathrm{SiO}_{2}$ layer was determined from spectroscopic ellipsometry measurements averaging over an area of about $1 \mathrm{~mm}$.

Periodic Au nanocluster arrays with a size of $10 \times 10 \mu \mathrm{m}^{2}$ were fabricated as reported previously [13] on $\mathrm{Si}$ and $75 \mathrm{~nm}$ thick $\mathrm{SiO}_{2}$ layers by direct electron beam writing (Raith-150, Germany) of a $130 \mathrm{~nm}$ spin-coated resist film (polymethyl methacrylate $950 \mathrm{~K}$ ). A $40 \mathrm{~nm}$ Au film with a $5 \mathrm{~nm}$ Ti underlayer for better adhesion was deposited by electron beam evaporation on the patterned positive resist. Finally, the resist was removed by a lift-off process in dimethylformamide resulting in a periodic array of Au nanoclusters. As a result, the fabricated SERS-active substrates consist of areas of Au nanoclusters with a period of $150 \mathrm{~nm}$.

CuS NCs were fabricated on the IERS and SERS substrates using the Langmuir-Blodgett technology as reported in [29]. Briefly, at the first stage behenic acid dissolved in hexane was spread onto the water surface in a $\mathrm{LB}$ bath using a $\mathrm{CuSO}_{4}$ solution as a subphase. The copper behenate films formed were then transferred (Y-type) from the water surface onto a bare silicon substrate or SERS-active substrates. The typical thickness of copper behenate films ranged from 200 to 4 monolayers (MLs) corresponding to an average $\mathrm{NC}$ film thickness from 33 to $0.7 \mathrm{~nm}$. At the second stage, the nucleation of CuS NCs in the organic matrix took place by sulfidizing the samples. At the last stage, $4 \mathrm{~h}$ of annealing at a temperature of $150{ }^{\circ} \mathrm{C}$ under argon atmosphere resulted in the removal of the organic matrix and the formation of free-standing $\mathrm{CuS}$ NCs on the substrates.

The morphology of the samples was determined using scanning electron microscopy (SEM). SEM images were obtained using a Raith-150 system at $10 \mathrm{kV}$ acceleration voltage, $30 \mu \mathrm{m}$ aperture and $6 \mathrm{~mm}$ working distance. It was found that the size of $\mathrm{Au}$ nanoclusters varied from array to array from 20 to $130 \mathrm{~nm}$, while the height is fixed to $55 \pm 5 \mathrm{~nm}$. CuS NCs have a spherical shape and an average size in the range of 5-8 $\mathrm{nm}$.

Raman experiments were carried out using Horiba T64000 and Labram HR800 spectrometers equipped with microscopes (the laser beam was focused to a spot with a size of $1 \mu \mathrm{m}^{2}$ ) in a backscattering geometry at room temperature. $\mathrm{He}-\mathrm{Cd}, \mathrm{Ar}^{+}$, DPSS Cobolt ${ }^{\circledR}$, and He-Ne lasers with wavelengths of 325 , $514.5,514.7$, and $632.8 \mathrm{~nm}$, respectively, and power below $2 \mathrm{~mW}$ were used for excitation.

\section{Acknowledgements}

The work was supported by the DFG project ZA146/22-1, DFG Research Unit 1713 "Sensoric Micro- and Nanosystems", 
Russian science foundation (project 14-12-01037), the cfAED cluster of excellence, by the Ministry of Education and Science of the Russian Federation, and CKP. Special thanks to K. P. Mogil'nikov for ellipsometry measurements of $\mathrm{SiO}_{2}$ layers with gradual thickness and determination of $\mathrm{SiO}_{2}$ thickness.

\section{References}

1. Honma, I.; Sano, T.; Komiyama, H. J. Phys. Chem. 1993, 97, 6692-6695. doi:10.1021/j100127a020

2. Milekhin, A. G.; Yeryukov, N. A.; Sveshnikova, L. L.; Duda, T. A.; Kosolobov, S. S.; Latyshev, A. V.; Surovtsev, N. V.; Adichtchev, S. V.; Himcinschi, C.; Zenkevich, E. I.; Jian, W.-B.; Zahn, D. R. T. J. Phys. Chem. C 2012, 116, 17164-17168. doi:10.1021/jp210720v

3. Hugall, J. T.; Baumberg, J. J.; Mahajan, S. Appl. Phys. Lett. 2009, 95, 141111. doi:10.1063/1.3243982

4. Chursanova, M. V.; Dzhagan, V. M.; Yukhymchuk, V. O.; Lytvyn, O. S.; Valakh, M. Ya.; Khodasevich, I. A.; Lehmann, D.; Zahn, D. R. T.; Waurisch, C.; Hickey, S. G. Nanoscale Res. Lett. 2009, 5, 403-409. doi:10.1007/s11671-009-9496-2

5. Lee, Y.-b.; Lee, S. H.; Lee, S.; Lee, H.; Kim, J.; Joo, J. Appl. Phys. Lett. 2013, 102, 033109. doi:10.1063/1.4788926

6. Todescato, F.; Minotto, A.; Signorini, R.; Jasieniak, J. J.; Bozio, R. ACS Nano 2013, 7, 6649-6657. doi:10.1021/nn402022z

7. Wang, X.; Kong, X.; Yu, Y.; Zhang, H. J. Phys. Chem. C 2007, 111, 3836-3841. doi:10.1021/jp064118z

8. Shan, G.; Xu, L.; Wang, G.; Liu, Y. J. Phys. Chem. C 2007, 111, 3290-3293. doi:10.1021/jp066070v

9. Liu, C. Y.; Dvoynenko, M. M.; Lai, M. Y.; Chan, T. H.; Lee, Y. R.; Wang, J.-K.; Wang, Y. L. Appl. Phys. Lett. 2010, 96, 033109 doi:10.1063/1.3291041

10. Milekhin, A. G.; Yeryukov, N. A.; Sveshnikova, L. L.; Duda, T. A.; Zenkevich, E. I.; Kosolobov, S. S.; Latyshev, A. V.; Himcinschi, C.; Surovtsev, N. V.; Adichtchev, S. V.; Feng, Z. C.; Wu, C. C.; Wuu, D. S.; Zahn, D. R. T. J. Exp. Theor. Phys. 2011, 113, 983-991. doi:10.1134/S1063776111140184

11. Rumyantseva, A.; Kostcheev, S.; Adam, P.-M.; Gaponenko, S. V.; Vaschenko, S. V.; Kulakovich, O. S.; Ramanenka, A. A.; Guzatov, D. V.; Korbutyak, D.; Dzhagan, V.; Stroyuk, A. L.; Shvalagin, V. V. ACS Nano 2013, 7, 3420-3426. doi:10.1021/nn400307a

12. Wang, Y.; Li, M.; Jia, H.; Song, W.; Han, X.; Zhang, J.; Yang, B.; Xu, W.; Zhao, B. Spectrochim. Acta, Part A 2006, 64, 101-105. doi:10.1016/j.saa.2005.07.003

13. Yeryukov, N. A.; Milekhin, A. G.; Sveshnikova, L. L.; Duda, T. A.; Rodyakina, E. E.; Sheremet, E. S.; Ludemann, M.; Latyshev, A. V.; Zahn, D. R. T. Thin Solid Films 2013, 543, 35-40. doi:10.1016/j.tsf.2013.03.070

14. Li, J.-H.; Chen, S.-W.; Chou, Y.; Wu, M.-C.; Hsueh, C.-H.; Su, W.-F. J. Phys. Chem. C 2011, 115, 24045-24053. doi:10.1021/jp203307f

15. Kahl, M.; Voges, E.; Kostrewa, S.; Viets, C.; Hill, W. Sens. Actuators, B 1998, 51, 285-291. doi:10.1016/S0925-4005(98)00219-6

16. Guillot, N.; Shen, H.; Frémaux, B.; Péron, O.; Rinnert, E.; Toury, T.; Lamy de la Chapelle, M. Appl. Phys. Lett. 2010, 97, 023113. doi:10.1063/1.3462068

17. Alvarez-Puebla, R.; Cui, B.; Bravo-Vasquez, J.-P.; Veres, T.; Fenniri, H. J. Phys. Chem. C 2007, 111, 6720-6723. doi:10.1021/jp070906s
18. Nemanich, R. J.; Tsai, C. C.; Connell, G. A. N. Phys. Rev. Lett. 1980, 44, 273-276. doi:10.1103/PhysRevLett.44.273

19. Ishii, M.; Shibata, K.; Nozaki, H. J. Solid State Chem. 1993, 105, 504-511. doi:10.1006/jssc.1993.1242

20. Yoon, D.; Moon, H.; Son, Y.-W.; Choi, J. S.; Park, B. H.; Cha, Y. H.; Kim, Y. D.; Cheong, H. Phys. Rev. B 2009, 80, 125422. doi:10.1103/PhysRevB.80.125422

21. Kolobov, A. V.; Maeda, Y.; Tanaka, K. J. Appl. Phys. 2000, 88, 3285-3289. doi:10.1063/1.1289818

22. Marsillac, S.; Little, S. A.; Collins, R. W. Thin Solid Films 2011, 519, 2936-2940. doi:10.1016/j.tsf.2010.11.065

23. Aspnes, D. E.; Theeten, J. B. J. Electrochem. Soc. 1980, 127, 1359-1365. doi:10.1149/1.2129899

24. Nemanich, R. J. Interference-enhanced Raman scattering from thin films and interfaces. In Microbeam Analysis; Russell, P. E., Ed.; San Francisco Press: San Francisco, CA, USA, 1989; pp 141-145.

25. Seok, T. J.; Jamshidi, A.; Kim, M.; Dhuey, S.; Lakhani, A.; Choo, H.; Schuck, P. J.; Cabrini, S.; Schwartzberg, A. M.; Bokor, J.; Yablonovitch, E.; Wu, M. C. Nano Lett. 2011, 11, 2606-2610. doi:10.1021/nl2010862

26. Min, Q.; Pang, Y.; Collins, D. J.; Kuklev, N. A.; Gottselig, K.; Steuerman, D. W.; Gordon, R. Opt. Express 2011, 19, 1648-1655. doi:10.1364/OE.19.001648

27. Gao, L.; Ren, W.; Liu, B.; Saito, R.; Wu, Z.-S.; Li, S.; Jiang, C.; Li, F.; Cheng, H.-M. ACS Nano 2009, 3, 933-939. doi:10.1021/nn8008799

28. Wang, D.; Zhu, W.; Best, M. D.; Camden, J. P.; Crozier, K. B. Nano Lett. 2013, 13, 2194-2198. doi:10.1021/nl400698w

29. Milekhin, A.; Sveshnikova, L.; Duda, T.; Surovtsev, N.; Adichtchev, S.; Ding, L.; Zahn, D. R. T. J. Vac. Sci. Technol., B 2010, 28, C5E22. doi:10.1116/1.3442799

\section{License and Terms}

This is an Open Access article under the terms of the Creative Commons Attribution License (http://creativecommons.org/licenses/by/2.0), which permits unrestricted use, distribution, and reproduction in any medium, provided the original work is properly cited.

The license is subject to the Beilstein Journal of Nanotechnology terms and conditions: (http://www.beilstein-journals.org/bjnano)

The definitive version of this article is the electronic one which can be found at: doi:10.3762/bjnano.6.77 